\title{
A Case of Burkholderia Prostatitis in a Patient with Chronic Granulomatous Disease
}

\author{
Sameer Bahal ${ }^{1}$ (D) Nisha Verma $^{1} \cdot$ Susan Tadros ${ }^{1} \cdot$ David M. Lowe $^{1} \cdot$ Siobhan O. Burns ${ }^{1,2}$
}

Received: 23 May 2020 / Accepted: 20 July 2020 / Published online: 16 September 2020

(C) Springer Science+Business Media, LLC, part of Springer Nature 2020

To the Editor,

Chronic Granulomatous Disease (CGD) is a rare, hereditary phagocytic immunodeficiency secondary to a defect in the NADPH (nicotinamide adenine dinucleotide phosphate) oxidase pathway leading to impaired superoxide formation [1]. The X-linked form is caused by a mutation in the CYBB gene. CGD patients are particularly susceptible to catalase positive bacterial and fungal infections with the lungs, skin, lymph nodes and liver being the most common sites of infection. Inflammatory complications are an additional source of morbidity with granulomatous inflammation commonly affecting the gastrointestinal and urinary tracts, skin and lungs. Antimicrobial, antifungal and steroid therapies remain the mainstay of conservative treatment for patients who have not undergone curative hematopoietic stem cell transplant or gene therapy.

Here, we report a case of prostatitis affecting a 34-year-old man who had been diagnosed with X-linked CGD in childhood. In the 4 weeks before the patient presented to our centre, he had had two short inpatient admissions to his local hospital with shortness of breath, cough and intermittent fevers. A CT scan of the chest and abdomen did not reveal a source of infection, and two nasopharyngeal swabs for SARS-CoV-2 infection were negative. No sputum sample for bacterial culture was obtained, but his symptoms partially improved with a course of co-amoxiclav. Towards the end of this illness, he developed pain affecting his back and abdomen. Less than a week later, the patient was readmitted to his local hospital with

Sameer Bahal and Nisha Verma are joint first author

Sameer Bahal

s.bahal@nhs.net

1 Department of Immunology, Royal Free Hospital, Pond Street, Hampstead, London, UK

2 Institute for Immunity and Transplantation, University College London, Pond Street, Hampstead, London, UK a 2-day history of fevers measuring up to $40{ }^{\circ} \mathrm{C}$, suprapubic and lower back pain, headaches and dysuria. There was no history of previous urinary tract infections, recent foreign travel or suspicion of a sexually transmitted cause. Of note, the patient had a past medical history of left testicular teratoma aged 24 treated with orchiectomy and 4 months of chemotherapy.

On the first day of admission, he did not pass urine, and urinary retention was suspected. Catheterization was attempted but abandoned due to severe pain. Elevated white cells were noted on urinalysis $\left(143 \times 10^{6} / \mathrm{L}\right.$ (reference range $\left.1-45 \times 10^{6} / \mathrm{L}\right)$ ); a provisional diagnosis of a urinary tract infection was made, and intravenous antibiotics were commenced.

After several days, he was transferred to our unit for continued management. C-reactive protein $(154 \mathrm{mg} / \mathrm{L}$, reference range $0-5)$ and prostate specific antigen $(7.94 \mathrm{mcg} / \mathrm{L}$, reference range $0-2.49$ ) on admission were both raised. White blood cell and neutrophil count and renal and liver function were normal. On palpation of the abdomen, there was significant suprapubic tenderness. A digital rectal examination elicited extreme discomfort on palpation of the prostate. An MRI scan performed on day 4 of the admission showed a $21 \mathrm{cc}$ prostate with diffuse signal enhancement (Fig. 1). No drainable prostatic abscess was seen. A diagnosis of prostatitis was made. An admission urine sample demonstrated 40-200 white blood cells per high-power field on microscopy, and culture yielded 1000-10,000 cfu/mL Burkholderia cepacia. The organism was sensitive to ceftazidime and cotrimoxazole and resistant to ciprofloxacin, ertapenem, temocillin and colistin. A subsequent post prostatic massage urine culture also confirmed this organism. The patient was treated with 7 days of intravenous meropenem $1 \mathrm{~g}$ TDS and teicoplanin $400 \mathrm{mg}$ BD. He also received oral prednisolone $40 \mathrm{mg}$ daily to prevent urinary obstruction related to local inflammation. On discharge, the patient's symptoms had largely resolved, and the CRP had fallen to $15 \mathrm{mg} / \mathrm{L}$. He completed a 6-week course of co-trimoxazole at home, and 

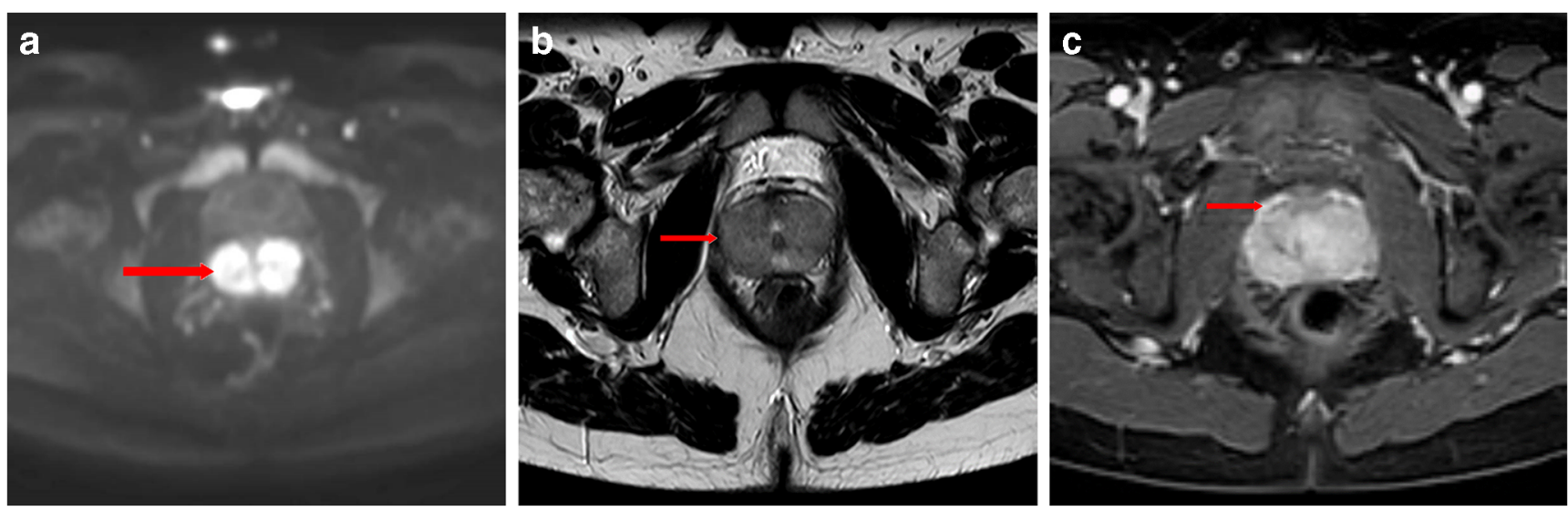

Fig. 1 MRI prostate scan images consistent with prostatitis. These show diffuse signal enhancement of the prostate on diffusion weighted images (a), loss of normal T2 high signal throughout most of the peripheral zone

steroids were weaned over 2 weeks. The patient had taken this antibiotic as infection prophylaxis, but its choice as a treatment agent was justified due to the higher dose and positive culture sensitivities. In addition, the patient had stopped cotrimoxazole prophylaxis while having taking co-amoxiclav in the weeks prior to admission to our unit.

Prostatitis in the general population is not age discrepant and can be acute or chronic. Gram-negative organisms such as Escherichia coli have been most commonly implicated in acute bacterial prostatitis, and chlamydia and gonorrhoea are frequent sexually transmitted causes. Burkholderia cepacia infection has also been reported though rarely and only as a complication of biopsy [2]. While prostatitis in CGD leading to hospitalization has not been described before, there have been 4 reported cases of prostatic abscesses. The first case involved a 15-year-old X-linked CGD patient who presented with unremitting cough and fevers and was treated for persistent left-sided pneumonia [3]. A baseline CT chest, abdomen and pelvis scan done to evaluate the extent of his disease identified a fluid collection in the prostatic fossa, later confirmed to be a prostatic abscess. Extensive microbiological analysis of the pus sample and urinalysis were negative except for a single colony of Candida albicans from the wound culture which was of uncertain significance. A CT-guided lung biopsy for persistent pneumonia, however, yielded Burkholderia cepacia responsive to meropenem and the patient clinically improved with this antibiotic. The second case was mentioned as part of a case series of 15 CGD patients with Serratia marcescens infection [4]. No specific clinical details were given. A third case occurred in a 35-year-old CGD patient who was treated with meropenem, vancomycin and rifampin after urine cultures grew a multidrug resistant Escherichia coli [5]. The final case involved a 41-year-old patient who developed a prostatic abscess detected after a whole body CT scan was performed for persistent fevers. Blood cultures grew Klebsiella pneumoniae, but sputum cultures were negative [6]. of the prostate (b), and hyper enhancement in the anterior portions of the peripheral zone as well as the adjacent transitional zone of the prostate on arterial phase images $(\mathbf{c})$. All lesions are marked with red arrows

In our case, prostatitis was preceded by a chest infection, and this presentation is similar to the first case of prostatic abscess described earlier. We hypothesize that in both cases, the lungs were the route of entry for the pathogen, and from there, it reached the prostate via the blood circulation.

We also considered the role that the previous history of testicular cancer played in the development of prostatitis. Although treatment with radiotherapy can be associated with prostatitis, the patient did not receive this and instead was managed with chemotherapy and orchiectomy. Therefore the remote history of testicular cancer may not have been relevant.

Our case report describes a rare complication of CGD and highlights the importance of investigating for other sources of infection aside from those commonly reported in this cohort, especially with symptoms' unresponsive to treatment or where initial investigations do not reveal a source of infection.

Data Sharing Data sharing is not applicable to this article as no datasets were generated or analysed.

Author Contributions SB, NV and ST wrote the article. DML and SOB reviewed the article. All authors were involved in treating the patient.

\section{Compliance with Ethical Standards}

Conflict of Interest The authors declare that they have no conflicts of interest.

Statement of Informed Consent The patient has provided written informed consent for the publication of this case report including the images.

\section{References}

1. Marciano BE, Spalding C, Fitzgerald A, Mann D, Brown T, Osgood $\mathrm{S}$, et al. Common severe infections in chronic granulomatous disease. Clin Infect Dis Off Publ Infect Dis Soc Am. 2015;60:1176-83.

2. Organ M, Grantmyre J, Hutchinson J. Burkholderia cepacia infection of the prostate caused by inoculation of contaminated ultrasound gel 
during transrectal biopsy of the prostate. Can Urol Assoc J. 2010;4: E58-60.

3. Agochukwu NQ, Rastinehad AR, Richter LA, Barak S, Zerbe CS, Holland SM, et al. Prostatic abscess in a pediatric patient with chronic granulomatous disease: report of a unique case and review of the literature. J Pediatr Surg. 2012;47:400-3.

4. Friend JC, Hilligoss DM, Marquesen M, Ulrick J, Estwick T, Turner ML, et al. Skin ulcers and disseminated abscesses are characteristic of Serratia marcescens infection in older patients with chronic granulomatous disease. J Allergy Clin Immunol. 2009;124:164-6.

5. Martini A, Katafigiotis I, Kalantzi S, Anastasiou I, Adamakis I, Toskas A, et al. Prostatic abscess in a patient with chronic granulomatous disease: a multi-disciplinary intervention. Urologia. 2017;84:267-9.

6. Wakabayashi Y, Jubishi D, Okamoto K, Ikeda M, Tatsuno K, Mizoguchi M, et al. A rare case of a prostatic abscess, bacteremia and chronic granulomatous disease associated with Klebsiella pneumoniae. J Infect Chemother Off J Jpn Soc Chemother. 2019;25:365-7.

Publisher's Note Springer Nature remains neutral with regard to jurisdictional claims in published maps and institutional affiliations. 